\title{
Habilidades motoras de crianças em berçários: estudo preliminar
}

\author{
Motor skills of children in nurseries: a preliminary study
}

Habilidades motoras de niños en viveros: estudio preliminar

\author{
Cinira Gomes ${ }^{1}$, Lislaine Cristina Dos Santos ${ }^{1}$, Lazaro Juliano Teixeira ${ }^{2}$, \\ Claudia Diehl Forti Bellani ${ }^{3}$
}

\begin{abstract}
1.Fisioterapeuta, Pós-graduada em Neurologia com ênfase em Neuropediatria (IBRATE). Balneário Camboriú-SC, Brasil.

2.Fisioterapeuta (FURB-SC), Mestre em Ciências (UNIFESP), Especialista em Fisioterapia Neurofuncional (ABRAFIN) e Pós-graduado em Fisioterapia em Neurologia (FURB). Balneário Camboriú-SC, Brasil.

3.Fisioterapeuta (ULBRA-RS), Mestre em Ciências Biológicas - Neurociências (UFRGS), Docente do Curso de Pós-graduação em Neurologia com ênfase em Neuropediatria da Faculdade de Tecnologia IBRATE. Balneário Camboriú-SC, Brasil.
\end{abstract}

\section{Resumo}

Objetivo. Comparar as habilidades motoras de deitar, rolar e sentar entre crianças frequentadoras de berçários da rede municipal e particular de Balneário Camboriú. Método. A amostra foi composta por 16 crianças ( 0 a 12 meses de idade), 8 de creches públicas e 8 de creche privada. Foi utilizado o instrumento de avaliação GMFM-Gross Motor Function Measure e aplicado aos responsáveis um questionário para analisar a situação socioeconômica das famílias. Utilizou-se a média e desvio padrão dos resultados em cada grupo, comparados entre si através da Diferença de Médias (DM) e Intervalos de Confiança (IC) de $95 \%$. Resultados. Observou-se que não houve diferença entre as médias do GMFM entre as crianças da creche pública $(61,33 \pm 10,18$ pontos) e da creche privada $(63,55 \pm 9,58$ pontos); DM = 2,22; IC de $95 \%$ de $-7,47$ a 11,91 pontos. As diferenças mais marcantes foram o grau de instrução do responsável (média de $+5,25$ de anos de estudo nos pais do setor privado, IC 95\% de 2,72 a 7,78 anos) e na renda familiar (média $+3,58$ salários mínimos no setor privado, IC $95 \%$ de 2,38 a 4,78). Conclusão. Preliminarmente, não foram encontradas diferenças marcantes no desenvolvimento motor entre crianças da rede pública e da rede privada em Balneário Camboriú.

Unitermos. pré-escolar; destreza motora; habilidades para a realização de testes; desenvolvimento infantil; deficiências do desenvolvimento

\begin{abstract}
Objective. To compare the motor skills of lying, rolling and sitting among children attending nurseries in the municipal and private schools in Balneário Camboriú. Method. The sample consisted of 16 children ( 0 to 12 months of age), 8 from public day care centers and 8 from private day care centers. The GMFM-Gross Motor Function Measure assessment instrument was used, and a questionnaire was applied to the parents to analyze the socioeconomic situation of the families. The mean and standard deviation of the results in each group were used, compared with each other through the Difference of Means (DM) and 95\% Confidence Intervals (CI). Results. There was no difference between the means of the GMFM between the children of the public daycare (61.33 \pm 10.18 points) and of the private daycare (63.55 \pm 9.58 points); $D M=2.22 ; 95 \%$ CI from -7.47 to 11.91 points. The most striking differences were the education level of the guardian (average of +5.25 years of schooling in parents in the private sector, 95\% CI from 2.72 to 7.78 years) and family income (average +3 , 58 minimum wages in the private sector, $95 \%$ CI from 2.38 to 4.78). Conclusion. Preliminarily, no striking differences in motor development were found between children from public and private schools in Balneário Camboriú.
\end{abstract}

Keywords. child preschool; motor skills; test taking skills; child development; developmental disabilities 


\begin{abstract}
Resumen
Introducción. El Alzheimer es una enfermedad neurológica degenerativa y progressiva, con prevalencia de 60 al 70\% de los casos de demencia diagnosticados. Objetivo. Describir las principales características de los ensayos clínicos de fisioterapia relacionados con la rehabilitación de pacientes con Alzheimer, publicados en la base de datos PEDro; clasificar, según el factor de impacto, las principales revistas que publican estudios en el área; y analizar la correlación entre calidad metodológica, factor de impacto y tiempo de publicación. Método. La búsqueda en la base de datos PEDro seleccionó ensayos clínicos que apuntaban a la rehabilitación de pacientes con Alzheimer, extrayendo datos como el idioma, el año y la hora de publicación, la calidad metodológica, el tamaño de la muestra, la intervención, la comparación y el factor de impacto de los periódicos. El coeficiente de correlación de Spearman se calculó para explorar las correlaciones entre las variables. Resultados. Encontramos 93 estudios publicados en 58 revistas. El número promedio de participantes por estudio fue de $102 \pm 155$ sujetos, la mayoría de ellos publicados en inglés ( $97 \%)$. La calificación promedio de Ios estudios PEDro fue de $5.5 \pm 1.5$. Ejercicios generales fueron el principal tipo de tratamiento utilizado $(43 \%)$, en comparación con otros tipos de intervención $(41 \%)$. El factor de impacto promedio de los periódicos fue de $6.7 \pm 11.6$. No se encontró correlación significante entre ninguna de las variables. Conclusión. Las publicaciones relacionadas con la rehabilitación de pacientes con Alzheimer presentando, en general, una calidad metodológica moderada. La mayoría de los estudios se publican en revistas con un factor de alto impacto (promedio 6,7 ). Finalmente, tanto los estudios actuales como los anteriores pueden tener una buena calidad metodológica, independientemente del factor de impacto de la revista.
\end{abstract}

Palabras clave. preescolar destreza motora; habilidades de prueba; desarrollo infantil; deficiencias de desarrollo

Trabalho realizado na Faculdade de Tecnologia IBRATE. Balneário Camboriú-SC, Brasil.

Conflito de interesse: não Recebido em: 2015 e re-enenviado em 2020

Aceito em: 08/04/2020

Endereço para correspondência: Lázaro J Teixeira. R. México, 875, Bairro das Nações, Balneário Camboriú-SC, Brasil. CEP 88338-223. E-mail: lazaroteixeira@gmail.com

\title{
INTRODUÇÃO
}

O desenvolvimento infantil nos seus primeiros anos de vida é caracterizado por constantes mudanças biológicas, psicossociais e emocionais, levando a importantes aquisições nos domínios motores, domínios sociais e cognitivos ${ }^{1}$. É na infância que ocorrem as maiores modificações físicas e psicológicas que caracterizam o crescimento e o desenvolvimento infantil. O desenvolvimento motor na infância inclui a aquisição de habilidades motoras, que possibilitam à criança a locomoção de diversas formas, 
trocas e aquisições posturais e manipulação de objetos diversos. Estas habilidades motoras adquiridas durante o desenvolvimento sofrem influência tanto de fatores pessoais como prematuridade, baixo peso, doenças genéticas ou fatores ambientais onde a criança está inserida, como um ambiente socioeconômico muito ruim, má nutrição e a qualidade do cuidado oferecido à criança².

Acreditava-se que as alterações no desenvolvimento motor resultavam apenas à maturação do sistema nervoso. Todavia, mais recentemente passou-se a acreditar que o comportamento motor pode ser influenciado de modo negativo ou positivo pelo ambiente que o sujeito está inserido ${ }^{3}$. Quando se fala em fatores ambientais deve-se considerar várias condições que influenciam 0 desenvolvimento de uma criança, como nutrição adequada, condições sociais favoráveis, ambiente familiar e educação infantil de qualidade ${ }^{4}$.

Muitas pesquisas relatam que o ambiente de educação infantil nem sempre age como promotor do desenvolvimento motor nos primeiros anos ${ }^{5}$. Logo, a qualidade das experiências vivenciadas pela criança e as interações com o meio ficam negligenciadas ${ }^{6}$. Desta forma, metas devem ser traçadas com o objetivo de abranger crianças de 0 a 6 anos de idade, priorizando o cuidado, o desenvolvimento de habilidades e a promoção de aprendizagem?.

Os prejuízos acarretados pelo atraso no desenvolvimento na primeira infância podem refletir posteriormente no desenvolvimento da criança através da 
predisposição à evasão escolar, gravidez na adolescência e delinquência juvenil ${ }^{1,8}$. Além disso, o fator familiar também interfere no processo de desenvolvimento da criança, principalmente a qualidade do vínculo mãe-filho. O poder aquisitivo das famílias já que com melhor renda a família tende a escolher creches com maior qualidade, adquirir brinquedos adequados e proporcionar um ambiente domiciliar que favoreça o desenvolvimento da criança.

Hoje sabe que muitas mães são responsáveis pelo sustento de suas casas e isso as leva a manter seus bebês em período integral nas escolas. A criança é inserida na creche a partir do terceiro mês de vida e volta ao convívio da família novamente ao final dia9 ${ }^{9}$. Logo, por ficar a maior parte do seu tempo na creche, o ambiente escolar será um fator de grande influência no desenvolvimento da criança.

Isso acarreta um número crescente de bebês em creches, tanto na rede pública quanto rede particular de ensino. Eis que surge à dúvida se a escolha entre uma creche pública ou privada interfere na evolução do desenvolvimento motor dessas crianças, já que, essas crianças passam a maior parte do dia longe do ambiente familiar e inserida no ambiente dos berçários.

O presente estudo teve como objetivo principal comparar as aquisições das habilidades motoras de deitar, rolar e sentar entre crianças frequentadoras de berçários da rede municipal e particular de Balneário Camboriú. Secundariamente, pretendeu-se identificar se eventuais 
atrasos na aquisição de habilidades motoras teriam relação com a condição sócio econômica dos pais.

\section{MÉTODO}

\section{Local do estudo}

A realização desta pesquisa foi aprovada pelo comitê de ética do Instituto Brasileiro de Terapias e Ensino - Faculdade Ibrate parecer 837.908. Todos os responsáveis pelos participantes do estudo assinaram o termo de consentimento livre e esclarecido. A coleta dos dados para a pesquisa foi realizada em três Núcleos de Educação Infantil vinculados à Secretaria de Educação de Balneário Camboriú (setor público) com autorização prévia através de Termo de Colaboração, e em um Centro de Educação Infantil (setor privado), que também autorizou a pesquisa.

\section{Amostra}

Os critérios de inclusão da pesquisa foram: as crianças deveriam frequentar a creche em período integral há pelo menos quatro meses e estarem aparentemente saudáveis. Na ocasião da avaliação, os critérios de exclusão do estudo foram crianças que apresentassem histórico de prematuridade, doença crônica ou recorrente, síndromes neurológicas ou qualquer deficiência (física, visual, auditiva já constatada. Além disso, as crianças que apresentassem choro, irritação ou febre no dia da avaliação também foram excluídas da amostra do estudo. 


\section{Procedimentos}

A avaliação das crianças foi feita após conferência dos critérios de inclusão pelas pesquisadoras, assinatura dos termos da pesquisa pelos responsáveis e devolução do questionário de avaliação socioeconômico familiar adequadamente preenchido.

O ambiente para avaliação foi à própria sala da aula, que foi preparada previamente conforme estrutura e disponibilidade de cada escola. Cada criança foi avaliada individualmente, durante uma única oportunidade por duas avaliadoras concomitantemente.

A duração de cada avaliação foi de aproximadamente de 30 a 40 minutos, e foram realizadas respeitando a rotina do berçário, como horários de alimentação, higiene e repouso. As avaliações foram realizadas no período vespertino, de setembro a novembro de 2014. Durante todo o período da avaliação uma professora ou uma auxiliar do berçário acompanhou o processo, com o objetivo de facilitar a familiarização da criança com as avaliadoras e auxiliar caso a criança apresentasse choro ou recusa.

O desfecho primário foi a presença das habilidades de deitar, rolar e sentar das crianças através do instrumento GMFM (Gross Motor Function Measure) ou Medida da Função Motora Grossa ${ }^{10}$, descrita através da média encontrada para cada grupo (entre 0 e $100 \%$ ). A GMFM é uma escala que busca quantificar a função e as mudanças nas atividades funcionais das crianças com paralisia cerebral e alguns 
outros problemas neurológicos através do tempo ${ }^{11}$. Pelo fato da GMFM utilizar os marcos para o desenvolvimento motor normal, os autores originais declaram que pode ser útil para outras crianças que não aquelas para as quais foi validada, mas sugerem que a confiabilidade e a validade devem ser estabelecidas ${ }^{10}$. Mesmo com estes estudos ainda não disponíveis no Brasil, é utilizada como padrão de avaliação em algumas instituições de referência ${ }^{11}$.

Este instrumento é composto por uma sequência de 88 itens, com descrição de movimentos agrupados em 5 dimensões (Dimensão A- deitar e rolar com 17 itens; Dimensão B - sentar com 20 itens; Dimensão C - engatinhar e ajoelhar com 14 itens; Dimensão D - em pé com 13 itens; Dimensão E - andar, correr e pular com 24 itens). Em cada item é atribuída à pontuação de 0 a 3 , sendo 0 quando não inicia o movimento, 1 quando inicia o movimento, 2 se completa parcialmente o movimento e 3 se completa 0 movimento. Tanto o resultado de cada dimensão, como o resultado final é apresentado por percentual entre 0 e $100 \%$ para cada dimensão, sendo $100 \%$ a medida ideal.

Tendo em vista que a idade esperada das crianças do berçário seria de crianças entre 5-8 meses, a previsão do projeto foi de utilizar apenas as Dimensões A (Deitar e Rolar) e B (Sentar) da GMFM, pois avaliam o desempenho de habilidades esperadas para esta idade, consideradas em uma análise de subgrupos do desfecho primário.

Como desfecho secundário foram considerados: a idade média das crianças em meses e a condição sócio econômica 
dos pais ou responsáveis, coletados através de questionário sócio econômico simples confeccionado pelas próprias pesquisadoras, com questões relacionadas à condição sócio econômica da família das crianças, como: escolaridade, renda, estado civil materno e questões sobre a estrutura familiar, cada item considerado em análise de subgrupo, sempre através da média encontrada nos dois grupos.

\section{Análise estatística}

A análise dos dados foi feita através dos grupos e subgrupos dos desfechos. Os achados dos desfechos foram apresentados através da média e do desvio padrão dos resultados das avaliações em cada grupo da amostra (Serviço Público X Serviço Privado).

Os achados foram comparados entre si através da diferença de média e do intervalo de confiança em cada desfecho (GMFM, idade e condição sócio econômica das famílias). A diferença de médias serve para comparar dados contínuos e representa a variação ocorrida quando a média e desvio padrão dos achados de um grupo é comparada com a média e desvio padrão do outro grupo, para cada desfecho e subgrupo de desfechos.

Assim, foram comparadas todas as médias entre 0 grupo de crianças do Setor Público com o grupo do Setor Privado. Se o resultado de diferença de médias resultou em um número positivo, significa que a média do segundo grupo (Privado) foi maior que a média do primeiro grupo (Público) e vice-versa. O intervalo de confiança de $95 \%$ é baseado no 
desvio padrão e apresenta a variação da tendência para aumentar ou diminuir a média comparada. Se o intervalo de confiança apresentar uma variação de um valor negativo até um valor positivo, interpreta-se a diferença entre as médias como não significante.

\section{RESULTADOS}

Participaram deste estudo 16 crianças de 0 a 12 meses de idade, de ambos os sexos, regularmente matriculadas em dois Núcleos de Educação Infantil (setor público) e em um Centro de Educação Infantil (setor privado) no município de Balneário Camboriú. Oito crianças frequentavam a rede pública e 8 crianças frequentavam a rede privada.

A Tabela 1 mostra os resultados dos escores (médias e desvios padrões - DP) da GMFM, da idade média dos grupos e do questionário sócio econômico encontradas nas duas amostras nos berçários públicos e privados, bem como as diferenças entre as médias encontradas em todas as variáveis pesquisadas e o intervalo de confiança de cada variável.

De acordo como desfecho primário (GMFM global) variou muito pouco entre os grupos. A pontuação média dos 8 indivíduos avaliados na creche pública, foi de $61,33 \pm 10,18 \%$ e dos 8 indivíduos da creche privada foi de $63,55 \pm 9,58 \%$. Isso significou que a diferença de médias (DM) encontrada foi de apenas 2,22 pontos, com um 
intervalo de confiança de 95\% (IC 95\%) que variou de $7,47 \%$ a $11,91 \%$, considerado não significante.

Tabela 1. Características dos participantes.

\begin{tabular}{|c|c|c|c|c|}
\hline & Público & Privado & & \\
\hline & Média $\pm D P$ & Média $\pm D P$ & $\begin{array}{l}\text { Diferença } \\
\text { de média }\end{array}$ & $\begin{array}{c}\text { Intervalo de } \\
\text { confiança } \\
(95 \%)\end{array}$ \\
\hline GMFM & $61,33 \pm 10,18$ & $63,55 \pm 9,58$ & 2,22 & $-7,47$ a 11,91 \\
\hline Dimensão $A$ & $62,24 \pm 14,36$ & $54,41 \pm 12,91$ & $-7,83$ & $-21,21$ a 5,55 \\
\hline Dimensão B & $60,41 \pm 15,58$ & $76,46 \pm 9,70$ & 16,05 & 3,33 a 28,77 \\
\hline $\begin{array}{l}\text { Média de idade da } \\
\text { criança (meses) }\end{array}$ & $8,88 \pm 1,46$ & $10,63 \pm 1,51$ & 1,75 & 0,29 a 3,31 \\
\hline $\begin{array}{l}\text { Características } \\
\text { socioeconômicas }\end{array}$ & & & & \\
\hline $\begin{array}{l}\text { Média de idade do } \\
\text { responsável (anos) }\end{array}$ & $25,50 \pm 4,99$ & $33,25 \pm 2,55$ & 7,75 & 3,87 a 11,63 \\
\hline $\begin{array}{l}\text { Grau de instrução dos } \\
\text { pais (anos de estudo) }\end{array}$ & $12,50 \pm 3,07$ & $17,75 \pm 1,98$ & 5,25 & 2,72 a 7,78 \\
\hline Pessoas na casa & $2,50 \pm 0,76$ & $2,25 \pm 0,46$ & $-0,25$ & $-0,87$ a 0,37 \\
\hline $\begin{array}{l}\text { Renda Familiar } \\
\text { (números de salário } \\
\text { mínimo) }\end{array}$ & $4,13 \pm 1,55$ & $7,71 \pm 0,76$ & 3,58 & 2,38 a 4,78 \\
\hline $\begin{array}{l}\text { Estado civil dos } \\
\text { responsáveis }\end{array}$ & $\mathbf{N}(\%)$ & $\mathbf{N}(\%)$ & & \\
\hline Casado & $4(50)$ & $8(100)$ & & \\
\hline Solteiro & $3(37,5)$ & & & \\
\hline Outros & $1(12,5)$ & & & \\
\hline
\end{tabular}

Em uma análise de subgrupos, observou-se que para a Dimensão A/Deitar e Rolar, também não apresentou diferença entre os grupos ( $D M=7,83 \%$ a menos no grupo da rede privada, IC $95 \%-21,21$ a 5,55$)$, de forma que todas as crianças possuíam as habilidades de deitar e rolar de forma equivalente, o que é previsível para a idade cronológica. 
$\mathrm{Na}$ Dimensão B/Sentar, verificou-se diferença de $16,05 \%$ a mais na amostra do setor privado, variável de $3,33 \%$ até $28,77 \%$ (IC 95\%), o que foi significante. O mesmo ocorreu no desfecho relativo à idade média das crianças. A média de idade no setor privado foi quase dois meses maior que a média de idade do setor público (DM=1,75 meses, IC 95\% de 0,29 a 3,31 meses). Estes achados são compatíveis entre si, pois espera-se que crianças com idade média de 10,5 tenham melhor desempenho da habilidade de sentar que crianças de 8,5 meses.

Observou-se também diferença em algumas características socioeconômicas entre os grupos, como a idade média do responsável, o grau de instrução do responsável e a renda familiar. No serviço privado, observou-se pais em média 7,75 anos mais velhos (IC 95\% de 3,87 a 11,63 anos), com uma média de 5,25 anos a mais de estudo (IC 95\% de 2,72 a 7,78 anos) e um rendimento mensal em média 3,58 salários mínimos a mais (IC $95 \%$ de 2,38 a 4,78).

Não houve diferença quanto ao número de pessoas em casa, sendo as famílias constituídas de 2,5 e 2,25 pessoas nas residências das crianças do serviço público e do privado, respectivamente. Todos os pais das crianças da rede privada eram casados, enquanto a metade dos pais das crianças do serviço público era casada, 37,5\% (3 indivíduos) solteiros e uma pessoa $(12,5 \%)$ assinalou outra situação. 


\section{DISCUSSÃO}

Os achados principiais desta pesquisa demonstraram não haver diferença significativa na aquisição de habilidades básicas do desenvolvimento motor entre crianças de creches públicas e privadas ao se avaliar a capacidade de deitar, rolar e sentar através da GMFM.

Ao se observar especificamente a Dimensão A/Rolar e Dimensão B/Sentar, não foi percebida diferença entre a dimensão A entre os grupos, de forma que todas as crianças possuíam as habilidades de deitar e rolar de forma equivalente, como previsto para a idade cronológica. Diferença foi observada na Dimensão B/Sentar, ocorreu possivelmente pela idade média das crianças da rede privada ter sido maior.

A coleta de dados iniciou nas creches públicas e estava programada para ser feita imediatamente em seguida na creche privada. Todavia, houve uma latência de um a dois meses entre a avaliação das duas, o que pode ter influenciado nos achados. Esta lacuna temporal se explica por demora de autorização dos locais de estudo e da agenda das avaliadoras.

Tendo em vista que a coleta de dados foi realizada mais tardiamente na creche do setor privado (entre 1-2 meses), acabou sendo realizada em crianças ligeiramente mais maduras e consequentemente, com um desenvolvimento motor ligeiramente mais avançado. Este fato levanta dúvida sobre a relevância destes achados preliminares e representou uma grande limitação deste estudo. 
Outros trabalhos com desenhos de estudo e propósitos semelhantes não encontraram diferenças entre os setores público e privado em relação ao desenvolvimento neuromotor de crianças. Faria et al. ${ }^{12}$, por exemplo, avaliaram e compararam o desenvolvimento neuromotor em pré-escolares de instituição pública e privada mostrando que não houve diferenças no desenvolvimento neuromotor. Havendo apenas diferenças em algumas habilidades mais que isso não acarretou prejuízos nos grupos de crianças avaliadas.

Estudos como este são fruto da curiosidade sobre a influência da creche no desenvolvimento infantil, uma vez que, no Brasil, com a inserção da mulher no mercado de trabalho, as creches tornaram-se um recurso indispensável para muitas famílias, sendo um local onde as crianças passam tempo integral.

A despeito da pequena diferença entre os achados do desenvolvimento das crianças neste estudo, possivelmente proporcionais à idade, outras diferenças que se destacaram estavam relacionadas com os fatores sociais das famílias. Em média, os pais das crianças em creches públicas eram 7,75 anos mais jovens, tinham em média 5,25 anos a menos de estudo e renda familiar média de 3,58 salários mínimos a menos que os pais das creches privadas.

O baixo nível econômico é um fator de risco para o desenvolvimento motor das crianças ${ }^{13,14}$. O nível econômico dos pais parece estar relacionado ao maior acesso à informação e, consequentemente, ao maior conhecimento a 
respeito dos mecanismos que podem gerar desenvolvimento motor mais adequado e ambiente estimulante aos filhos ${ }^{14}$.

Além disso, sabe-se da importância que tem a escolaridade materna para o desenvolvimento dos filhos por interferir diretamente na qualidade do estímulo doméstico. O maior grau de escolaridade da mãe está associado a melhores oportunidades de estímulos ambientais. Tal resultado parece ser explicado pelo fato de as mães com maior grau de instrução terem renda mais elevada, maior acesso as informações e melhor conhecimento sobre o processo de desenvolvimento de seus filhos ${ }^{14}$.

Os desfechos com maior diferença neste estudo preliminar foram: a renda, o grau de instrução e a idade média dos responsáveis, que aparentemente, podem não ter refletido nas aquisições motoras dos filhos. Outros autores já evidenciaram ausência de relação entre a condição socioeconômica dos pais e o desempenho motor da criança.

Baltieri et al. ${ }^{9}$ em seus estudos sobre desempenho motor de lactentes frequentadores de creche encontraram achados semelhantes aos desta pesquisa e não identificou relação direta entre a escolaridade dos pais e condições socioeconômicas com a aquisição motora das crianças avaliadas.

Souza et al.15 também não observaram influência de fatores de risco biológico (como a prematuridade) e fatores de risco ambiental (como espaço físico onde a criança vive, escolaridade dos pais, nível de instrução da mãe e a renda familiar) com o desempenho das crianças nascidas a termo 
e a pré-termo, o que também subsidia os achados deste trabalho.

Logo, por ficar a maior parte do seu tempo na creche, presume-se que o ambiente escolar pode ser um fator de grande influência no desenvolvimento da criança e que, pelos achados preliminares, foi possível supor que, tanto a creche particular quanto a pública, são ambientes favoráveis para o desenvolvimento global dos seus participantes. Entretanto, mais dados são necessários para refutar ou confirmar esta hipótese.

É importante a estimulação para o processo de neuroplasticidade nas crianças, que inicia no período embrionário e termina da vida extrauterina ${ }^{16}$.

Quando falamos de neuroplasticidade entende-se por qualquer modificação do sistema nervoso que não seja periódico e que tenha duração maior que poucos segundos, ou ainda, a capacidade de adaptação do sistema nervoso, especialmente a dos neurônios, as mudanças nas condições do ambiente que ocorrem no dia a dia dos indivíduos. Esse processo é maior durante a infância e declina gradativamente, sem se extinguir na vida adulta ${ }^{17}$. Assim as experiências familiares e ambientais se relacionam com o desenvolvimento global das crianças e essas interações, quando vivenciadas, estimulam a capacidade de neuroplasticidade em função dessas experiências ${ }^{18}$.

Desta forma, é importante detectar precocemente fatores ambientais que prejudiquem ou favoreçam os processos de desenvolvimento da criança. Identificado o 
problema acredita-se que há necessidade de ações que envolvam os profissionais de educação, saúde e a própria família, prevenindo futuros déficits e assim favorecer $o$ desempenho escolar, motor e os relacionamentos sociais e familiares.

\section{CONCLUSÃO}

Conforme as variáveis pesquisadas, observamos que, em nenhum dos ambientes pesquisados, as crianças apresentaram atrasos nas habilidades motoras globais avaliadas, e que, aparentemente, não houve diferença no desenvolvimento motor de indivíduos menores de um ano matriculados em escolas públicas e privadas no município de Balneário Camboriú.

Os fatores que apresentaram maior diferença entre os grupos avaliados foram: a renda familiar e o nível de escolaridade dos pais. Todavia, estes fatores aparentemente não interferiram na aquisição das habilidades motoras das crianças. Ou seja, os pais das crianças do berçário público apresentaram uma renda familiar mais baixa e também escolaridade inferior aos pais das crianças do berçário privado, mas isto talvez não determine que as crianças apresentem atraso do desenvolvimento.

Os achados deste estudo preliminar justificam 0 aprofundamento da pesquisa que investiguem, não somente o ambiente escolar, mas também o correlacionem com o ambiente domiciliar, por meio de amostras maiores. 


\section{REFERÊNCIAS}

1.Santos MM, Corsi C. Comparação de desempenho motor e cognitivo de crianças freqüentadoras de creches públicas e particulares. Braz J Phys Ther 2013;17:579-87. http://dx.doi.org/10.1590/S141335552012005000126

2. Martinello M, Nazario PF, Marques LZ, Ries LGK, Lando C. Avaliação do desenvolvimento motor de crianças matriculadas em creches públicas. HU Revista 2011;37:155-62.

https://periodicos.ufjf.br/index.php/hurevista/article/view/1326

3. Hallal CZ, Marques NR, Bracialli LMP. Aquisição de habilidades funcionais na área de mobilidade em crianças atendidas em um programa de estimulação precoce. Rev Bras Crescimento Desenvol Hum 2008;18:27-34.

http://pepsic.bvsalud.org/scielo.php?script=sci arttext\&pid=S010412822008000100005

4.Spessato BC, Valentini NC, Krebs RJ, Berleze A. Educação infantil e intervenção motora: um olhar a partir da teoria bioecológica de Bronfenbrenner. Movimento Rev Edu Fis UFRGS 2009;15:147-73. https://seer.ufrgs.br/Movimento/article/view/5617/6951

5.Souza CT, Santos DCC, Tolocka RE, Baltieri L, Gibim NC, Habechian FAP. Avaliação do desempenho motor global e em habilidades motoras axiais e apendiculares de lactentes frequentadores de creche. Rev Bras Fisioter 2010;14:309-15. http://dx.doi.org/10.1590/S1413$\underline{35552010000400007}$

6. Almeida C, Valentini N. Contexto dos berçários e um programa de intervenção no desenvolvimento de bebês. Motricidade 2013;9:22-32. http://dx.doi.org/10.6063/motricidade.9(4).744

7.Pereira LCL, Grave MQ. Encaminhamento de crianças com necessidades educacionais especiais em idade de estimulação precoce a escolas de Educação Infantil de um município de médio porte do Vale dos Sinos. Rev Educ Espec 2012;25:101-14. http://dx.doi.org/10.5902/1984686X2820

8. Franco V, Melo M, Apolónio A. Problemas do desenvolvimento infantil e intervenção precoce. Educar em Revista 2012:49-64. http://dx.doi.org/10.1590/S0104-40602012000100005

9.Baltieri L, Santos DCC, Gibim NC, Souza CT, Batistella ACT, Tolocka RE. Desempenho motor de lactentes frequentadores de berçários em creches públicas. Rev Paul Pediatr 2010;28:283-9. http://dx.doi.org/10.1590/S0103-05822010000300005

10.Russel DJ, Rosenbaum PL, Avery LM, Lane M. Medida da Função Motora Grossa (GMFM-66 \& GMFM-88): Manual do usuário. São Paulo: Memnon; 2011.

11.Nunes LCBG. Tradução e Validação de Instrumentos de Avaliação Motora e de Qualidade de vida em Paralisia Cerebral (Tese). Campinas: Universidade Estadual de Campinas; 2008. http://taurus.unicamp.br/bitstream/REPOSIP/260635/1/Nunes LigiaC hristinaBorsatoGuimaraes D.pdf 
12.Faria TCC, Sousa L, Silva JRT, Borges JBC, Parisi JR. Comparação da avaliação neuromotora em pré-escolares de instituição pública e privada. Rev Neurocienc 2015;23:248-54.

https://doi.org/10.4181/RNC.2015.23.02.1013.7p

13.Amorim RCA, Laurentino GEC, Barros KMFT, Ferreira ALPR, Filho AGM, Raposo MCF. Programa de saúde da família: proposta para identificação de fatores de risco para o desenvolvimento neuropsicomotor. Rev Bras Fisioter 2009;13:506-13. http://dx.doi.org/10.1590/S1413-35552009005000065 14.Defilipo ÉC, Frônio JS, Teixeira MTB, Leite ICG, Bastos RR, Vieira MT, et al. Oportunidades do ambiente domiciliar para 0 desenvolvimento motor. Rev Saúde Pública 2012;46:633-41. http://dx.doi.org/10.1590/S0034-89102012005000040

15.Souza ES, Magalhães LC. Desenvolvimento motor e funcional em crianças nascidas pré-termo e a termo: influência de fatores de risco biológico e ambiental. Rev Paul Pediatr 2012;30:462-70. http://dx.doi.org/10.1590/S0103-05822012000400002

16. Oliveira CEN, Salina ME, Annunciato NF. Fatores Ambientais que influenciam a plasticidade do SNC. Acta Fisiátriaca 2001;8:6-13. http://www.revistas.usp.br/actafisiatrica/article/view/102269/100647 17. Borella MP, Sacchelli T. Os efeitos da prática de atividades motoras sobre a neuroplasticidade. Rev Neurocienc 2009;17:161-9. https://doi.org/10.34024/rnc.2009.v17.8577

18.Silva MFMC, Kleinhans ACS. Processos cognitivos e plasticidades cerebral da Síndrome de Down. Rev Bras Ed Esp 2006;12:123-38.

https://doi.org/10.1590/S1413-65382006000100009 